\title{
Simple motor performance of patients with Parkinson's disease before and after a surgical lesion in the thalamus
}

\author{
ETIENNE PERRET ${ }^{1}$ \\ From the Montreal Neurological Institute, McGill University, \\ Montreal, Canada
}

Studies of motor behaviour in patients with Parkinson's disease, and of its modification by a stereotaxic operation for the relief of tremor and/or rigidity have yielded somewhat inconsistent results (Schwab, Chafetz, and Walker, 1954; Petrinovich and Hardyck 1964; Talland and Schwab, 1964). For instance, Bélanger and Saunier (1961) have found a purely contralateral beneficial effect of a stereotaxic operation in a simple tapping task, whereas Meier, Story, French, and Chou (1966) have reported that a post-operative improvement in tapping performance occurs in the preferred hand only, regardless of the side of the surgical lesion. Several factors have led to these discrepancies, the most important of which is probably the location of the surgical lesion. Thus, the various studies have been carried out to investigate the effects on motor functions of lesions in the globus pallidus, thalamus, or subthalamus. Even though these placements may bear similar relationships to the tremor and rigidity observed clinically, it seems probable that other aspects of motor behaviour will be differentially affected by different localizations of lesion. The variety of neurosurgical techniques used to make the lesions (mechanical, electrical, chemical) further complicates the problem. Finally, the many different objective and quantitative measures of motor function which have been applied are another important cause of this heterogeneity of results. The tests used range in complexity from simple measures of the strength of grip (Bélanger and Saunier, 1961) to the simultaneous performance of two different tasks (Talland and Schwab, 1964). King (1959) and Talland (1963) have studied the effects of Parkinson's disease on various components of motor behaviour by modifying one aspect of a basic motor task at a time. Such an approach

\footnotetext{
${ }^{1}$ This investigation was carried out while the author held a fellowship awarded by the Swiss National Science Foundation.

Present address: Neurochirugische Universitätsklinik, 8006 Zurich Switzerland.
}

enables one to isolate more specific effects of sur- $\frac{\Omega}{01}$ gical lesions on motor behaviour than the mere $s$ abolition of the clinical symptoms of tremor and $\overrightarrow{0}$ rigidity. Furthermore, the difference between the $\overrightarrow{-}$ results of Bélanger and Saunier (1961) and those of $\omega_{\sigma}$ Meier et al. (1966) lays stress on the necessity of considering cerebral dominance for motor functions $\frac{\bar{O}}{0}$ as a possible specific factor in the modification of $\dot{\omega}$ motor behaviour by a stereotaxic operation.

The present investigation has been undertaken in to find out if Parkinson's disease and the subsequeit $\stackrel{\infty}{\oplus}$ stereotaxic operation have specific differentiक्ञा음 effects on the motor performance of the preferreand non-preferred hands, and also on different aspects of one simple motor task.

METHODS

A tapping task subdivided into several subtasks was used, such that a certain number of different factors underlying motor performance could be isolated. Four monomanual and two bimanual tapping subtasks were so devised, that one motor component (amplitude of movement) and the degree of sensorimotor coordination $\stackrel{2}{\rightarrow}$ (precision of movement) could be varied independently of each other. The tapping task as a whole was kept at a fairly low level of complexity, to rule out the uncontrollable effect of pre-existing differences in the manualo skill of the subjects. In addition, the strength of grip was measured in all subjects in order to get some baseline on simple muscular function.

SUBJECTS Patients Seventeen right-handed patients with Parkinson's disease were tested one or two days 0 before, and seven to eight days after a unilateral stereo- $₹$ taxic operation for the relief of tremor and/or rigidity. 윽 Nine patients-five men and four women - underwent $a_{\supset}$ left-sided operation (LSO), and eight patients-five meno and three women-underwent a right-sided operation (RSO). The two patient groups proved to be well-matched $\mathcal{N}$ for age, duration of the Parkinsonian symptoms, and $N$ pre-operative intelligence quotient, as assessed with Form I of the Wechsler-Bellevue Intelligence Scale (Table I).W 
TABLE I

AGE, DURATION OF PARKINSONIAN SYMPTOMS, AND PREOPERATIVE I.Q. OF THE TWO PATIENT GROUPS

\begin{aligned} \multicolumn{1}{l}{ LSO-group } & $R S O$-group \\ \hline Mean $\pm S . D &$. Mean $\pm S . D \\$. \hline $58.4(6.1) & 56.6(5.7) \\ 8.8(6.9) & 7.4(7.2) \\ 115.3(13.3) & 116.0(9.4) \\ 112.0(13.7) & 112.5(8.2)\end{aligned}$

The stereotaxic operations were performed by Dr. Gilles Bertrand. The target was the nucleus ventralis lateralis of the thalamus, and the lesions were placed mechanically with a leucotome (Bertrand, Blundell, and Musella, 1965).

Control subjects Four right-handed normal adultstwo men and two women-matched for age with the patients, served as control subjects. Their mean age was 53.3 years (S.D. \pm 8.6 years). They were tested twice, with the same time-interval between test-sessions as the patients. In spite of their small number, there is no reason to reject the validity of their scores, none of which differed significantly from those of a larger but younger normal control group.

APPARATUS Strength of grip A Collins Hand Dynamometer was used for this measurement.

Tapping task The apparatus is shown in Figure 1. On one of the two boards, three small horizontal metal plates $(1,2$, and 3$), 3 \mathrm{~cm}$ in diameter, were screwed at a distance of $28 \mathrm{~cm}$ from each other. On the second board, large vertical metal plates (A, B, and C), $13 \mathrm{~cm}$ high by $10 \mathrm{~cm}$ wide, and facing each other, were fixed also at a distance of $28 \mathrm{~cm}$ from each other. The middle plate (B) could be easily removed from the board. All plates were connected electrically in series, with a $6 \mathrm{~V}$ d.c. power supply, and from there, with two electro-mechanical counters and two styluses. Touching any of the plates with one stylus would, therefore, close the electrical circuit and activate the corresponding electro-mechanical counter.
PROCEDURE Strength of grip At the beginning of each test-session, two dynamometric readings were made alternately for each hand, starting with the right hand.

Tapping task The six tapping subtasks were performed next. The subject held one stylus in each hand. Each trial lasted for $10 \mathrm{sec}$. The order was as follows:

a. Precise tapping over a small distance Starting at the centre (plate 2 in Fig. 1), the two adjoining small plates had to be tapped alternately as fast as possible with one stylus, thus requiring a to-and-fro movement of one hand (plates 2, 3, 2, etc. for the right hand, and plates 2, 1, 2, etc. for the left hand). In order to hit the small plates a high degree of sensorimotor coordination was required. Two trials were done alternately with each hand, starting with the right hand.

b. Precise tapping over a large distance Same as (a), but plates 1 and 3 were used for each hand, thus doubling the amplitude of movement as compared with subtask (a). The right hand started at plate 1, the left hand at plate 3 .

c. Gross tapping over a small distance Same as (a), but using the large vertical plates and starting at the middle one-that is, the right hand had to hit plates B and C alternately (Fig. 1) as fast as possible, and the left hand had to hit plates B and A. In this subtask, therefore, a gross to-and-fro movement of one hand was required. Because the plates were so large and were placed vertically, only a low degree of sensorimotor coordination was necessary.

d. Gross tapping over a large distance Same as (c), but using plates $\mathbf{A}$ and $\mathbf{C}$ for each hand (plate $\mathrm{B}$ being removed), thus simply doubling the amplitude of movement as compared with subtask $(c)$.

e. Symmetrical bimanual tapping Both hands had to tap the small plates simultaneously. The right hand tapped plates 2 and 3 (Fig. 1), and at the same time, the left hand tapped plates 2 and 1 . The subject thus performed symmetrical movements with his two hands. Only seven patients of each patient group and the four control subjects did this subtask.

f. Parallel bimanual tapping Same as subtask (e), except that the right hand tapped plates 2 and 3 at the same time

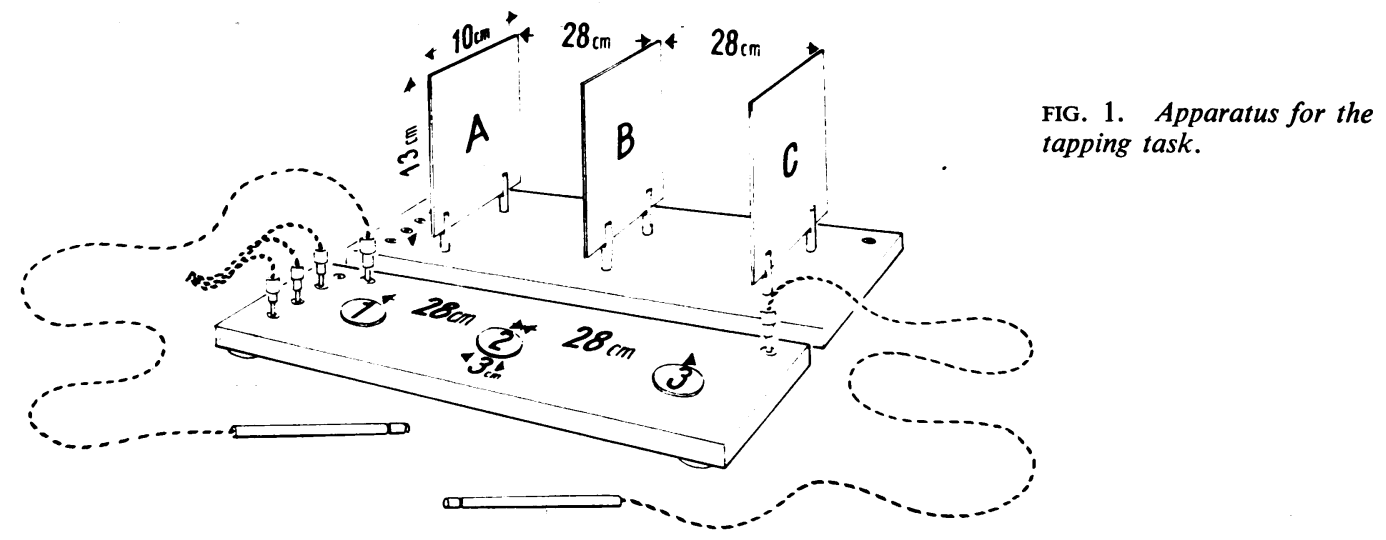


as the left hand tapped plates 1 and 2, thus producing parallel movements of the two hands. Only seven patients of each patient group and the four control subjects did this subtask.

Subtasks $(e)$ and $(f)$ were done twice each in alternation.

SCORING The dynamometric readings were in $\mathbf{k g}$, and the average of two readings for each hand was analysed statistically.

The scores for the tapping subtasks were the number of contacts/10 sec. As the individual scores did not show any learning effect, the average of the two trials for each hand was calculated in the four monomanual subtasks. In the two bimanual subtasks, the number of simultaneous contacts/10 sec was read, and the average of the two trials was calculated for each subtask.

ANALYSIS OF RESUlTS IN THE TAPPING TASK Precision in tapping The average scores in the two subtasks using the small plates (subtasks $a$ and $b$ ) were compared with the average scores of the two subtasks using the large plates (subtasks $c$ and $d$ ). This procedure eliminated the effect of the amplitude of movement-that is, average precise tapping performance and average gross tapping performance were compared with each other.

Amplitude of movement in tapping The average scores in the two subtasks requiring a small movement (subtasks $a$ and $c$ ) were compared with the average scores in the two subtasks requiring large movements (subtasks $b$ and $d$ ). This eliminated the effect of the precision of movement-that is, the average tapping performance over a small distance was compared with the average tapping performance over a large distance.

Bimanual tapping The average scores in subtasks $(e)$ (symmetrical tapping) and $(f)$ (parallel tapping) were compared in a single statistical analysis with the average score of the left hand in subtask (a) (precise monomanual tapping over a small distance).

\section{RESULTS}

PRE-OPERATIVE TESTING The pre-operative scores which reflect the influence of Parkinson's disease are summarized in Tables II and III, showing the means (M) and the standard deviations (S.D.) for the strength of grip and for the tapping performance ${ }^{\circ}$ respectively.

Strength of grip The analysis of variance, based $\stackrel{\frac{\rho}{3}}{\frac{3}{2}}$ on the average dynamometric readings, yields a significant groups $\times$ hands interaction $(P<0.01)$. The control group is somewhat stronger with the $\underset{\vec{S}}{\vec{S}}$ right hand than with the left. In the LSO group both hands have equal strength, and in the RSO $\frac{}{0}$ group the right hand is much stronger than the left $\frac{\bar{\omega}}{2}$ (Table II). These differences between hands in the $\widetilde{\Phi}$ patient groups were expected. They merely show that the hand more affected by the Parkinsonian ${ }^{\infty}$ symptoms is relatively weaker than the less affected $\vec{O}$ hand.

Precision in tapping The analysis of variance $\vec{\omega}$ comparing the precise and gross tapping performance shows a significant difference between the subject groups $(P<0.01)$. Both patient groups tap much more slowly than the control group (Tabte $\vec{\omega}$ III).

TABLE II

STRENGTH OF GRIP: PRE-OPERATIVE RESULTS (MEANS ( AND STANDARD DEVIATIONS (S.D.) IN KG)

\begin{tabular}{|c|c|c|c|}
\hline & TANDARD D & TIONS (S.D & J KG) \\
\hline & LSO & RSO & Control \\
\hline & $M \pm S . D$. & $M \pm S . D$. & $M \pm S . D$. \\
\hline $\begin{array}{l}\text { Right hand } \\
\text { Left hand }\end{array}$ & $\begin{array}{l}24 \cdot 2(20 \cdot 6) \\
24 \cdot 0(21 \cdot 9)\end{array}$ & $\begin{array}{l}37 \cdot 3(16 \cdot 0) \\
22 \cdot 8(14 \cdot 7)\end{array}$ & $\begin{array}{l}45.0(13 \cdot 7) \\
42.2(16.0)\end{array}$ \\
\hline
\end{tabular}

TABLE III

TAPPING TASK: PRE-OPERATIVE RESUltS (MEANS (M) AND STANDARD DEVIATIONS (S.D.) IN CONTACTS/10 SEC)

\begin{tabular}{|c|c|c|c|c|c|}
\hline LSO-group & & RSO-group & & Control-gro & \\
\hline $\begin{array}{l}\text { Right hand } \\
M \pm S . D .\end{array}$ & $\begin{array}{l}\text { Left hand } \\
M \pm S . D .\end{array}$ & $\begin{array}{l}\text { Right hand } \\
M \pm S . D .\end{array}$ & $\begin{array}{l}\text { Left hand } \\
M \pm S . D .\end{array}$ & $\begin{array}{l}\text { Right hand } \\
M \pm S . D .\end{array}$ & $\begin{array}{l}\text { Left hand } \\
M \pm S . D .\end{array}$ \\
\hline
\end{tabular}

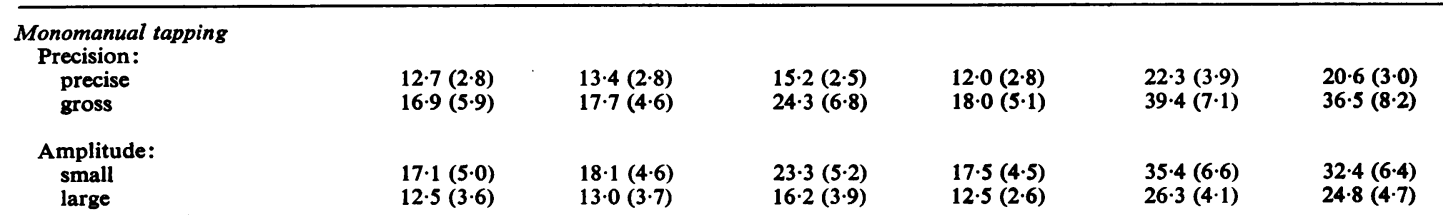

Bimanual tapping

Symmetrical

Parallel

$\frac{M \pm S . D}{10.4(3.0)}$

$\frac{M \pm S . D}{10 \cdot 7(2 \cdot 9)}$

$\frac{M \pm S . D .}{19.5(5.6)}$

Monomanual control

(small plates

small amplitude)

$(14 \cdot 9(3 \cdot 3))$

$(13 \cdot 4(2 \cdot 6))$

$(23 \cdot 0(3 \cdot 7))$ 
The groups $\times$ hands interaction is also significant $(P<0 \cdot 01)$. The control group taps faster with the right hand than with the left. In the patient groups, the significant interaction indicates that the hand less affected by Parkinsonian symptoms performs relatively faster than does the more affected hand. The lateralization of the Parkinsonian symptoms and the effect of right-handedness are cumulative

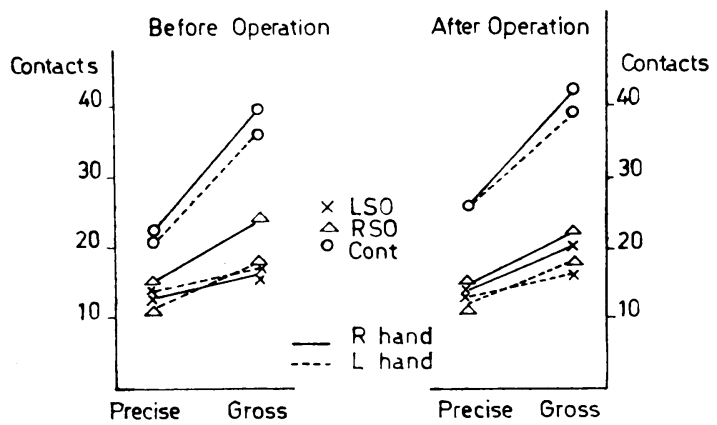

FIG. 2. Pre-and post-operative performance in precise and gross monomanual tapping.

in the RSO group, whereas they compensate each other in the LSO group.

The groups $\times$ precision interaction is significant $(P<0.01)$. This is the statistical expression of the fan-like arrangement of lines connecting the precise with the gross tapping scores in Fig. 2, for each group and regardless of the hand tested.

Amplitude of movement in tapping The analysis of variance comparing the performance when large or small movements have to be made, shows a significant difference between the groups of subjects $(P<0.01)$, which is due to the control subjects tapping faster than the patients (Table III).

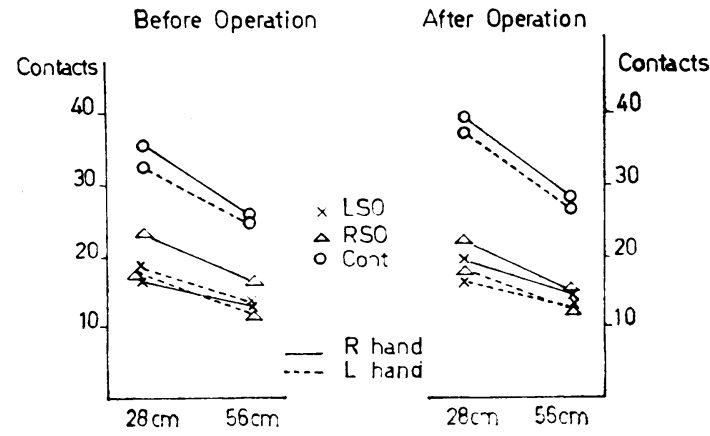

FIG. 3. Pre- and post-operative monomanual tapping performance with the small $(28 \mathrm{~cm})$ and large $(56 \mathrm{~cm})$ amplitudes of movement.
The groups $\times$ hands interaction is significant $(P<0.01)$, showing again that the hand which is less affected by Parkinsonian symptoms taps relatively faster than the more affected hand.

The groups $\times$ amplitude of movement interaction is significant $(P<0.01)$, due to the fan-like arrangement of lines connecting the average performance over the small and the large amplitudes in Fig. 3, for each group of subjects and regardless of the right and left hands.

Bimanual tapping The only statistically significant difference is between the groups of subjects $(P<0.01)$. Again, the control subjects tap much faster than the patients, and the patient groups are not different from each other (Table III). In addition, tapping with one hand allows a better performance than tapping bimanually, but this difference does not reach statistical significance. Neither does the difference between symmetrical and parallel tapping reach significance in any of the three groups of subjects.

POST-OPERATIVE CHANGES The post-operative scores have been summarized in Table IV for the strength

TABLE IV

STRENGTH OF GRIP: POST-OPERATIVE RESULTS (MEANS (M) AND STANDARD DEVIATIONS (S.D.) IN KG)

\begin{tabular}{|c|c|c|c|}
\hline & LSO & $R S O$ & Control \\
\hline & $\overline{M \pm S . D .}$ & $\overrightarrow{M \pm S . D .}$ & $\overline{M \pm S . D}$ \\
\hline $\begin{array}{l}\text { Right hand } \\
\text { Left hand }\end{array}$ & $\begin{array}{l}25.2(18.7) \\
21.4(19.0)\end{array}$ & $\begin{array}{l}32 \cdot 1(15 \cdot 2) \\
25 \cdot 6(16 \cdot 0)\end{array}$ & $\begin{array}{l}44 \cdot 8(16 \cdot 3) \\
42 \cdot 4(13 \cdot 5)\end{array}$ \\
\hline
\end{tabular}

of grip, and in Table $\mathrm{V}$ for the tapping performance. The effects of a stereotaxic operation, however, are reflected by the post-operative changes in performance. These will be considered next, expressed as a percentage of the pre-operative performance.

Strength of grip There is no test-retest change in the control group, as shown in Table VI. In both patient groups there is an increase in the strength of grip in the hand contralateral to the side of the surgical lesion, and a decrease in the ipsilateral hand, but these differences are not significant.

Precision in tapping The test-retest differences in the control group indicate a practice effect which is similar for both hands, and greater in precise than in gross tapping. In the LSO group there is a clear post-operative improvement in the right hand and a slight decrease of performance in the left hand. The post-operative changes of both hands differ almost significantly in precise tapping $(P<0 \cdot 10)$, and significantly in gross tapping $(P<0.05)$. In the RSO group there is a slight post-operative improvement of tapping performance in the left hand, and 
TABLE V

TAPPING TASK: POST-OPERATIVE RESULTS (MEANS (M) AND STANDARD DEVIATIONS (S.D.) IN CONTACTS/10 SEC).

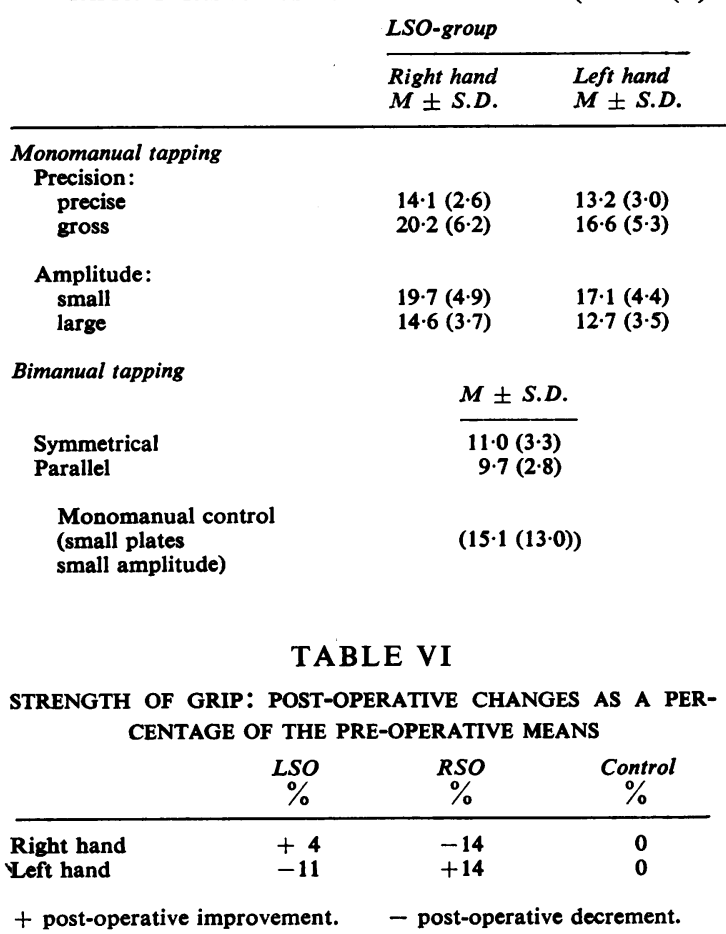

TABLE VII

TAPPING TASK. POST-OPERATIVE CHANGES AS A PERCENTAGE OF THE PRE-OPERATIVE MEANS

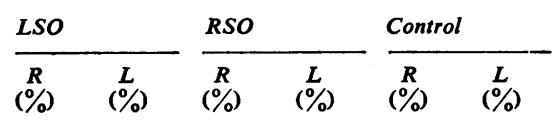

\begin{tabular}{lllllll}
\hline $\begin{array}{c}\text { Precision: } \\
\text { precise }\end{array}$ & +11 & -2 & -3 & +5 & +18 & +24 \\
gross & +20 & -6 & -7 & +2 & +7 & +7 \\
& & & & & & \\
$\begin{array}{c}\text { Amplitude: } \\
\text { small }\end{array}$ & +15 & -6 & -4 & +4 & +12 & +16 \\
large & +18 & -2 & -6 & +1 & +10 & +8
\end{tabular}

Bimanual :

symmetrical $+6 \quad-15 \quad+12$

parallel $-2 \quad-17 \quad+13$

R: Right hand. L: Left hand.

+ post-operative improvement.

- post-operative decrement.

a slight decrease in the right hand, but the postoperative changes of both hands do not differ significantly (Table VII).

Amplitude of movement in tapping The postoperative changes are essentially the same as for precision in tapping (Table VII). In the control group the test-retest improvement of performance

\begin{tabular}{ll} 
RSO-group & \\
\hline Right hand & Left hand \\
$M \pm S . D$. & $M \pm S . D$.
\end{tabular}

Control-group

Right hand Left hand

$M \pm S . D . \quad M \pm S . D$.

\begin{tabular}{|c|c|c|c|}
\hline $\begin{array}{l}14.8(3.7) \\
22.6(8.2)\end{array}$ & $\begin{array}{l}12.6(2.5) \\
18.4(5.7)\end{array}$ & $\begin{array}{l}26 \cdot 1(4 \cdot 3) \\
42 \cdot 3(10 \cdot 6)\end{array}$ & $\begin{array}{l}25.5(3.0) \\
38.9(8.2)\end{array}$ \\
\hline $\begin{array}{l}22 \cdot 3(6 \cdot 2) \\
15 \cdot 2(5 \cdot 6)\end{array}$ & $\begin{array}{l}18.2(4.9) \\
12.6(3.5)\end{array}$ & $\begin{array}{l}39.6(8 \cdot 1) \\
28.8(6.3)\end{array}$ & $\begin{array}{l}37.6(7.0) \\
26.8(5.7)\end{array}$ \\
\hline
\end{tabular}

$$
\frac{M \pm S . D .}{9 \cdot 1(2 \cdot 0)}
$$$$
\frac{M \pm S . D .}{21 \cdot 8(6 \cdot 7)}
$$$$
(13 \cdot 7(3 \cdot 2))
$$

$(28 \cdot 8(4 \cdot 8))$

is similar for both hands. In the LSO group there is a marked post-operative improvement in performance for the right hand, but a slight decrease for the left hand. The post-operative changes of both hands differ significantly for the smats amplitude of movement only $(P<0.05)$. In the RSQ group there is a slight improvement of tapping performance in the left hand, and a slight decrease in the right hand, but the difference between these changes is not significant.

Bimanual tapping In the second test-session the control subjects tap faster than in the first, whether symmetrical or parallel movements are required. In the LSO group the post-operative changes are very small, but they are in the direction of an improvement of bimanual performance. In the RSO group on the contrary, there is a clear, though nonsignificant, decrease of bimanual tapping performance in symmetrical as well as in parallel tapping.

\section{DISCUSSION}

The purpose of this investigation was to analyse the effects of Parkinson's disease, and of a stereotaxically-placed, unilateral thalamic lesion upon certain measurable aspects of simple motor performance. The results obtained before operation, which reflect the influence of Parkinson's disease, will be considered before discussing the postoperative changes in performance, which show the effects of a superimposed thalamic lesion on simple motor performance.

PRE-OPERATIVE RESULTS Parkinson's disease does not affect the performance of the preferred and $N$ 
non-preferred hands differentially-at least, not for the measures sampled here. Thus, the preoperative results need to be considered only in terms of hand more affected versus hand less affected by the Parkinsonian symptoms. The differences in the performance of the two hands are similar for the strength of grip and for the monomanual tapping subtasks, although completely different aspects of motor behaviour are involved in both cases; Parkinson's disease lowers mainly the performance of the hand more affected by tremor and rigidity. An additional indication for the lack of a specific relation between Parkinson's disease and hand preference is given by the results in the bimanual tapping subtasks. In these subtasks, where a global performance is obtained regardless of the separate performance of each hand, the results are the same in both patient groups, but indicate impairment relative to the normal control group.

The different aspects of motor behaviour studied here are not affected differentially by Parkinson's disease. Both doubling the amplitude and increasing the precision of movement produce a performance decrement which is proportionally the same in all subject groups. King (1959) has produced a linearly increasing difference between the performance of patients with Parkinson's disease and control subjects, by adding successively a simple movement, then self-initiation of the signal, and finally a coordinative movement (shifting an object from one point to another) to a basic simple reaction time task. King's results, therefore, are in agreement with the present ones, in that they also show the broad spectrum of the detrimental effects of Parkinson's disease on motor behaviour.

POST-OPERATIVE CHANGeS The comparison of the post-operative and pre-operative scores shows different effects of unilateral thalamic lesions, depending on whether the hand contralateral or the hand ipsilateral to the side of the lesion is considered, and also depending upon pre-existing hand preference.

First, the performance of the hand contralateral to the side of the lesion improves after surgery. In the monomanual subtasks this improvement is clearly stronger in the LSO group than in the RSO group. In other words, a surgical lesion in the cerebral hemisphere dominant for motor functions produces more improvement in the contralateral hand than a lesion in the non-dominant hemisphere. The neuroanatomical and neurophysiological mechanisms underlying this finding are not known, and the most likely explanation seems not to be directly related to cerebral dominance as such. The abolition of tremor and rigidity in the preferred hand may lead to the more intensive use of that hand immediately after the operation, because the preferred hand is the most useful for the patient when functioning normally. In constrast, the abolition of tremor and rigidity in the non-preferred hand is less important for many practical activities of daily life. However, this hypothesis is not confirmed by the results of Meier et al. (1966), who reported an improvement of tapping performance always in the preferred hand, regardless of the side of a subthalamic lesion. Yet Bélanger and Saunier (1961) found an equal improvement in the contralateral hand whether a lesion in the globus pallidus was made on the right or left side. In these two studies the tapping tasks used were similar to two of the tapping subtasks devised for the present investigation, so that the discrepancies in the results cannot be attributed to the mere structure of the tasks. Differences in procedure may however partly account for the discrepancies. For instance, in Meier et al.'s study (1966) the test trials lasted for $\mathbf{3 0}$ seconds, which may have introduced a fatigue effect in the performance of their patients. Bélanger and Saunier (1961) probably reduced the influence of hand preference by giving four trials of practice for each hand before measuring the performance. Yet the most obvious difference in the three studies lies in the location of the surgical lesions. The question arises, then, as to whether lesions in the globus pallidus, thalamus, and subthalamus are differentially related to cerebral dominance for motor functions, perhaps enhancing it in the case of the thalamus and subthalamus, and perhaps counterbalancing it in the case of the globus pallidus.

The second point is related to the unexpected finding of this study: a stereotaxic operation produces a decrement of performance, albeit small, in the hand ipsilateral to the side of the surgical lesion. The hypothesis is that one hand being more affected by Parkinsonian symptoms before the operation, the less affected hand has to adapt to the other in order to reach the best possible coordination of movement. In this case, the more affected hand directly benefits from an operation, whereas the other hand is not influenced directly, but becomes unbalanced by the sudden change in the formerly more affected hand. If this is so, it must be expected that the decrement of performance in the ipsilateral hand will be only transient.

The third point concerns bimanual tapping. A slight overall post-operative improvement of performance is observed in the LSO group and a clear, though statistically non-significant, decrement of performance in the RSO group. The hypothesis formulated for the ipsilateral decrement in monomanual tapping may also apply here, but it must be 
completed by taking into account the cerebral dominance for motor functions. Sudden unbalancing of the non-preferred hand by a surgical lesion in the dominant hemisphere would have no adverse effect on the bimanual coordination of movement, whereas unbalancing the preferred hand by a lesion in the non-dominant hemisphere would affect bimanual coordination adversely. It must be noted that only the unbalancing of one hand as such seems to produce an effect specifically related to cerebral dominance, since both patient groups performed equally well in bimanual tapping before the operation.

Concerning the differential effects of a stereotaxic operation on the two aspects of motor behaviour studied here-namely, the amplitude and the precision of movement, the present results are negative. A unilateral thalamic lesion seems to affect motor behaviour as a whole, as does Parkinson's disease.

\section{SUMMARY}

Monomanual and bimanual tapping performance have been studied in 17 patients with Parkinson's disease, before and after a stereotaxic operation (nucleus ventralis lateralis of the thalamus). The results indicate that, in simple monomanual tapping, Parkinson's disease depresses performance mainly in the hand more affected by the symptoms, regard- less of hand preference. A surgical lesion improves $\underline{\overline{0}}$ performance in the formerly more affected hand, $Z$ especially if this is the preferred hand, but tends to depress performance in the other hand. In simplee bimanual tapping, performance remains unchanged after an operation in the dominant hemisphere, and it decreases after an operation in the non-dominant hemisphere.

I am grateful to Dr. Gilles Bertrand for the opportunity to test his patients, and to Dr. Brenda Milner for her constant and most valuable advice.

\section{REFERENCES}

Bélanger, D., and Saunier, M. (1961). Etude analytique des processus $\overline{\mathcal{S}}$ psychophysiologiques dans la maladie de Parkinson. Rev. canad. Biol., 20, 539-544.

Bertrand, G., Blundell, J., and Musella, R. (1965). Electrical exploration of the internal capsule and neighbouring structures during stereotaxic procedures. J. Neurosurg., 22, 333-343.

King, H. E. (1959). Defective psychomotor movement in Parkinson's disease: Exploratory observations. Percept. Mot. Skills, $9, \vec{\omega}$ 326.

Meier, M. J., Story, J., French, L. A., and Chou, S. N. (1966). Quantitative assessment of behavioral changes following subthalamotomy in the treatment of Parkinson's disease. Confin. neurol. (Basel), 27, 154-161.

Petrinovich, L., and Hardyck, C. (1964). Behavioral changes in Parkinson patients following surgery. J. chron. Dis., $1, \omega$ 225-233.

Schwab, R. S., Chafetz, M. E., and Walker, S. (1954). Control of trin simultaneous voluntary motor acts in normals and in Parkf sonism. Arch. Neurol. Psychiat. (Chic.)., 72, 591-598.

Talland, G. A. (1963). Manual skill in Parkinson's disease. Geriatriç 18, 613-620.

, and Schwab, R. S. (1964). Performance with multiple sets Parkinson's disease. Neuropsychologia, 2, 45-53. 